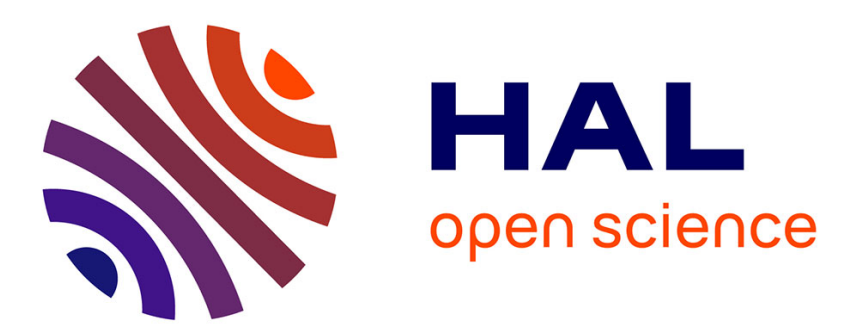

\title{
A New High-Current Large-Bandwidth DC Active Current Probe for Power Electronics Measurements
}

\author{
Patrick Poulichet, F. Costa, E. Laboure
}

\section{To cite this version:}

Patrick Poulichet, F. Costa, E. Laboure. A New High-Current Large-Bandwidth DC Active Current Probe for Power Electronics Measurements. IEEE Transactions on Industrial Electronics, 2005, 52 (1), pp.243-254. 10.1109/tie.2004.841066 . hal-01870526

\section{HAL Id: hal-01870526 \\ https://hal.science/hal-01870526}

Submitted on 7 Jan 2020

HAL is a multi-disciplinary open access archive for the deposit and dissemination of scientific research documents, whether they are published or not. The documents may come from teaching and research institutions in France or abroad, or from public or private research centers.
L'archive ouverte pluridisciplinaire HAL, est destinée au dépôt et à la diffusion de documents scientifiques de niveau recherche, publiés ou non, émanant des établissements d'enseignement et de recherche français ou étrangers, des laboratoires publics ou privés. 


\title{
A NEW HIGH CURRENT, LARGE BANDWIDTH DC ACTIVE CURRENT PROBE FOR POWER ELECTRONICS MEASUREMENTS
}

\author{
P. Poulichet ${ }^{1}$, F. Costa ${ }^{2}$ Member IEEE, E. Labouré ${ }^{2}$ \\ ${ }^{1} \mathrm{ESIEE}$, \\ 2 Bd Blaise Pascal \\ 93162 Noisy le Grand, France. Fax. 33 1.45.92.66.99 \\ patrick.poulichet@esiee.fr \\ ${ }^{2}$ SATIE, ENS de Cachan \\ 61 Avenue du Président Wilson \\ 94230 Cachan, France. 33 1. 45.92.21.99 \\ Francois.COSTA@satie.ens-cachan.fr, Eric.LABOURE@satie.ens-cachan.fr
}

\begin{abstract}
This paper is focused on the design and the realization of two high current probes (up to $100 \mathrm{~A}$ ) whose bandwidths range from DC to $50 \mathrm{MHz}$. These performances have been met by the association of an active Hall sensor and a passive current transformer. This association will be named "active current transformer" (ACT). It will be shown that it has better frequency performances than the classical closed-loop current transducer, usually used for high current measurements but frequency limited. The EMI issues, due to the sensor electrical environment are respected thanks to the shielded structure and special inner arrangements of the Hall sensors. Because of its large bandwidth, its large current ranging and its EMI ruggedness, this current probe is well matched to power electronics measurements.
\end{abstract}

Key words: Current probe, Hall sensor, Current transformer, EMI, power electronics.

\section{INTRODUCTION}

The area of power electronics requires high performance current probes for the following reasons:

- Static converter's switching frequency extends from $100 \mathrm{~Hz}$ to $1 \mathrm{MHz}$, so high cut-off frequency of current transformers has to be extended to over $10 \mathrm{MHz}$.

- Current shape can be sinusoidal or pulsed containing a DC component or high frequency ringings.

- MOSFET or IGBT are generating high $\mathrm{dI} / \mathrm{dt}$; values up to several $\mathrm{kA} / \mu \mathrm{s}$ are usually reached. So, static converters generate strong radiated and conducted EMI [5]-[6].

These specific performances are difficult to achieved in a single type of current sensor [7]. Our purpose herein is to develop a DC to $50 \mathrm{MHz}$ current probe that is well adapted to these constraints. The high frequency behavior of a current transformer has been studied in [8]. The alteration of the high frequency response is deeply affected by the primary conductor position, by an air gap in the core and the presence of a shielding case around the transformer extend the high cut-off frequency. In our probe, a current transformer is used in order to measure the high frequency components of current and, at low frequency, a special arrangement of Hall sensor is used. To correct the linking between the two frequency domains, an optimized correcting network is used.

This work is organized as follows. Section II reminds the operating principle of a classical closed-loop current transducer and its limitations. Section III presents the working principle of the single stage ACT and its modeling. The new double stage ACT (ACT ${ }^{2}$ ) is depicted in section IV and its performances are compared to the single stage one. Some design considerations will be highlighted. Experimental results in time and frequency domains will be presented and compared to theoretical analysis throughout all the paper. 


\section{Closed-loop Hall-effect current transducers}

The closed-loop Hall effect current transducer is well matched to medium performances and economical applications [1], [11], [12]. The operating principle is reminded in Fig. 1.a: The primary ampere-turns are compensated by the secondary ampere-turns supplied by an amplifier whose input signal is delivered by a Hall sensor situated in the air gap. The system is balanced when the flux in the air-gap is zeroed, so DC and AC currents can be measured. The low sensitivity and the temperature drift of the Hall sensor are corrected by the closedloop structure.
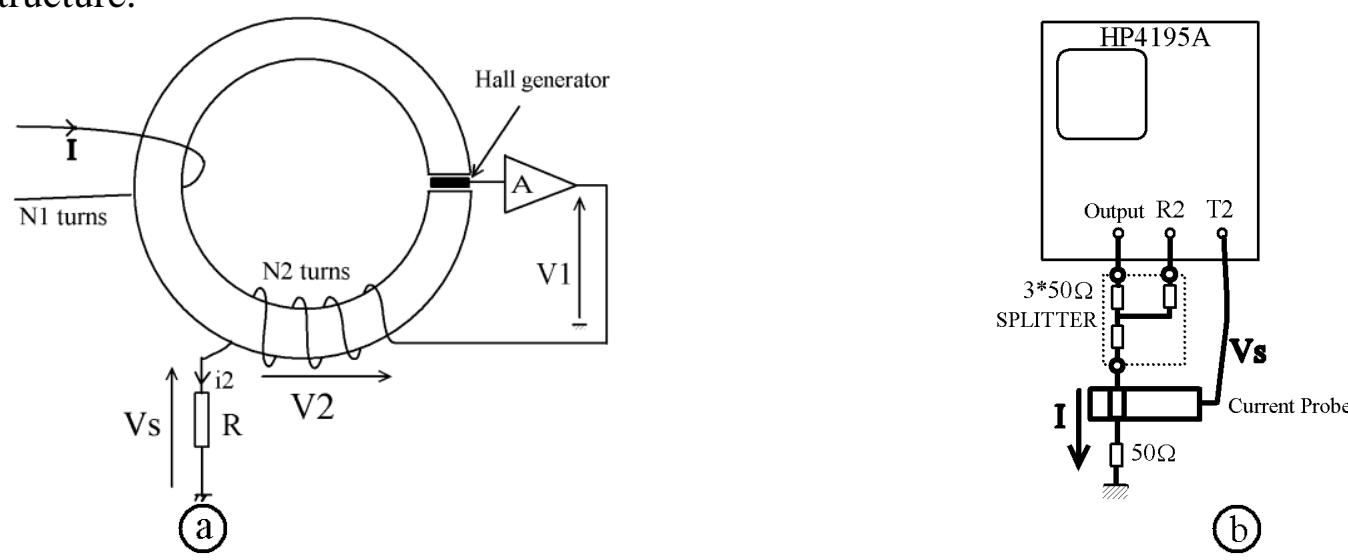

Fig. 1: (a) Operating principle of the closed-loop Hall effect current transducer. (b) Gain Phase measurement of current probe.

The limitations of the performances are mainly due to the amplifier and secondarily due to the Hall sensor. In the case of a large current gauge, the amplifier has to deliver a significant output current. It is known that the bandwidth of an amplifier is limited when its output current increases. Consequently, a 100 A device is limited to few tenth of $\mathrm{kHz}$ [1]. A solution would be to increase the secondary turns number, but the winding resistance increases as well as the parasitic capacitive effects and the stray inductance. The increase of the winding resistance leads to increase the supply voltage of the amplifier in order to balance the voltage drop. Therefore, a trade-off has to be find between the secondary turns number, the amplifier output current and the useful bandwidth of the transducer. Practically, the figure-of-merit of these devices (product current gauge $\mathrm{x}$ bandwidth) is limited around $10^{7} \mathrm{~A} . \mathrm{Hz}$.

Secondly, the dynamic limitations of the amplifier generates a magnetic saturation if a high $\mathrm{dI} / \mathrm{dt}$ current is applied to the primary. Afterward, the core remains polarized and an offset voltage appears at the output of the amplifier.

A third kind of limitations is due to the Hall element. Its sensitivity depends on temperature and on its polarizing current. These two parameters have to be electronically controlled, leading to increase the cost of the sensor. Some semiconductors manufacturers propose integrated Hall sensors, embedding the amplifier and the correction circuits [2], [10]. In this way, the sensitivity is guaranteed.

The frequency operating domain of a Hall-effect current transducer can be separated in two parts:

- in DC and at low frequency, only the Hall sensor delivers a signal while the voltage induced at the secondary of the transformer is near zero.

- at high frequency, the flux is around zero in the magnetic core and the output of the Hall sensor too. The output voltage is induced mainly by the secondary winding.

In the intermediate operating frequency domain, the two effects are superimposed, and the frequency response of the current transducer isn't flat as shown in Fig. 2 for commercial 
products. The transfer function (Sensitivity Vs/I versus frequency) is measured using Fig. 1.b measurement diagram. Before any measurement, the output current is calibrated by measuring voltage at the output of the splitter with input T2. The maximum value of current through the probe is around $6 \mathrm{~mA}$. The transfer function of a commercial product [1] exhibits a $20 \%$ trough in the transfer curve around $40 \mathrm{kHz}$.

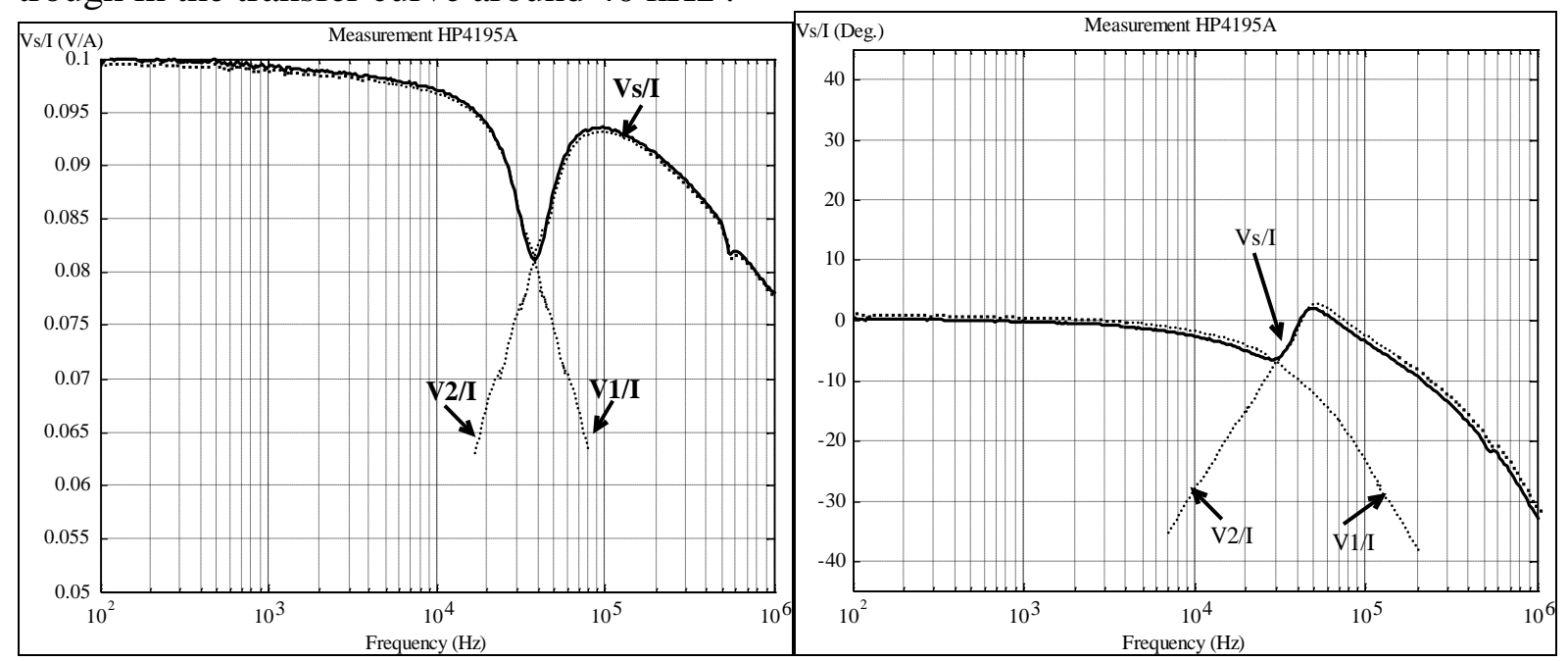

Fig. 2: Typical transfer function of a current transducer.

So, in order to improve the figure-of-merit of the high-gauge current sensor and to overcome the previous drawbacks, we propose a solution that associates an open-loop Hall sensor and a current transformer as depicted in the following sections.

\section{Single stage Active Current Transformer (ACT)}

\section{A. Principle}

This principle has still be used in some performing commercial products [4]. It associates in series a Hall sensor and a current transformer. The realization is depicted in Fig. 3.a and the resulting bandwidth is shown in Fig. 3.b. We suppose that all the devices are linear. At low frequency, only the Hall sensor delivers the ACT voltage while at high frequency the output ACT voltage is essentially delivered by the secondary winding of the transformer. The output voltage can be expressed by (1):

$$
\mathrm{V}_{\mathrm{s}}=\mathrm{V}_{\mathrm{amp}}+\mathrm{V}_{\text {tran }}
$$

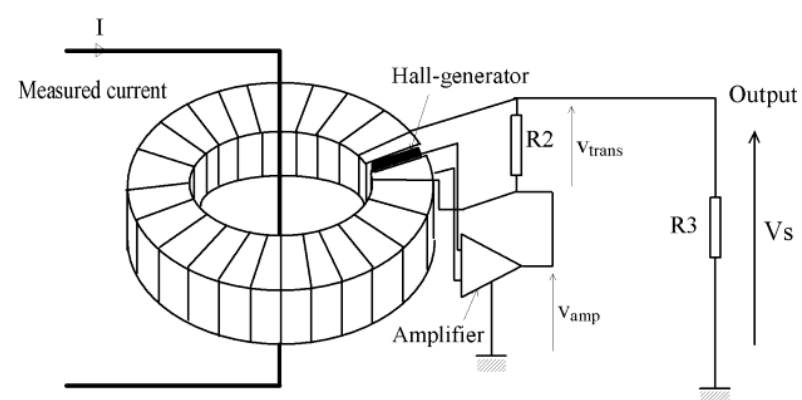

(a)

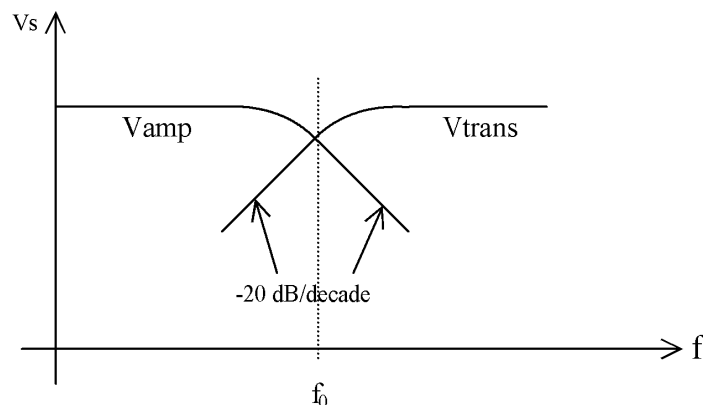

(b)

Fig. 3: (a) Operating principle of open-loop Hall. (b) Transfer function. 
The slope is $-20 \mathrm{~dB} / \mathrm{decade}$ for the Hall sensor and $+20 \mathrm{~dB} / \mathrm{decade}$ for the transformer. That means that frequency response would be flat from DC up to high frequency. Practically, that is not the case due to the current flowing through resistance $\mathrm{R}_{3}$. However, the resulting trough in the bandwidth can be corrected, as depicted in the following sections.

At low frequency, sensitivity is adjusted by the gain of the amplifier. At high frequency, the sensitivity depends on the secondary to primary turns ratio and on the $R_{2} / / R_{3}$ resistance value. Resistance $R_{3}$ is connected at the output of the coaxial cable in order to avoid wavereflections.

The main advantage of this structure is due to its open-loop behavior. Actually, the amplifier has just to deliver the load current through $\mathrm{R}_{3}$, that remains small compared to the one required in closed-loop current transducers. Consequently, the frequency limitation is less restrictive, and the figure-of-merit value can be increased significantly. However, the drawback compared to open-loop structure is that the scattering of Hall sensor sensitivity is no longer balanced.

\section{B. Practical design considerations}

We have realized a $100 \mathrm{~A}, \mathrm{DC}$ to $30 \mathrm{MHz}$ ACT. Its sensitivity factor is $100 \mathrm{mV} / \mathrm{A}$, and the device is shielded to respect EMI issues. The diagram of the ACT is shown in Fig. 4.a. Actually, two Hall sensors IC1 and IC2 (SS495 from Honeywell) have been used to improve the EMI ruggedness at low frequency. An integrated and fully trimmed device has been selected in our application in order to increase the sensitivity and to keep the accuracy better than $1 \%$ [2]. The Hall sensors have been associated symmetrically in differential mode and connected to the instrumentation amplifier (IC3 AD 620) in order to reject the common mode perturbations. At the outputs of the Hall sensors, two low-pass filters (R1, R3, C1 and R2, R4, C2) are connected to reduce the high frequency noise.

A FT40 Zn-Mn ferrite ring core has been chosen because of its good frequency performances (outer diameter $40 \mathrm{~mm}$, inner diameter $20 \mathrm{~mm}$, height $15 \mathrm{~mm}$ ). A $2 \mathrm{~mm}$ air-gap avoids the magnetic core to be saturated and increases the linearity.

The buffer IC8 (BUF 634) is used as a current amplifier with constant output resistance. This resistance will be taken into account in the calculus of sensitivity. For the rated 100 A current in the primary, the output amplifier must delivered $0.2 \mathrm{~A}\left(\mathrm{R}_{2} / / \mathrm{R}_{3}=10 / 50\right)$. A dipole is placed at the outputs of the transformer to correct the frequency trough as explained later.

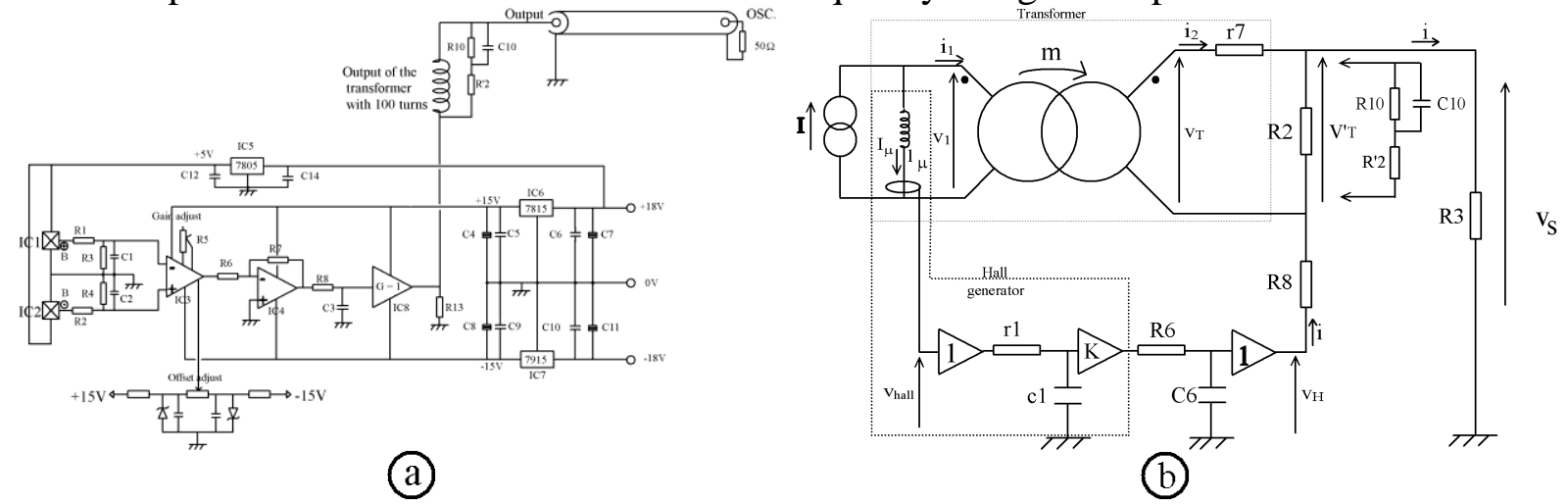

Fig. 4 : (a) Diagram of the ACT. (b) Model of the ACT.

\section{ACT modeling and trough cancellation}

In order to correct the frequency trough, the sensitivity of the transformer is increased in a particular frequency range. This is done by replacing resistance $\mathrm{R}_{2}$ of Fig. 3.a by the 
correcting dipole constituted by $\mathrm{R}_{2}, \mathrm{R}_{10}$ and $\mathrm{C}_{10}$ of Fig. 4.b: At low frequency, the output voltage of the transformer is increased thanks to resistance $R_{2}{ }_{2}+R_{10}$. Over the cut-off frequency determined by $\mathrm{R}_{10}$ and $\mathrm{C}_{10}$, the sensitivity is reduced. The best values for $\mathrm{R}_{2}, \mathrm{R}_{10}$ and $\mathrm{C}_{10}$ are determined owing to an optimization algorithm [3] which uses a Matlab® minimization algorithm and which works over the full frequency range. This method leads to an accuracy around $1 \%$.

The model that is used to calculate the transfer function of the ACT and its correcting network is shown in Fig. 4.b. The parasitic capacitances and the leakage inductances are not included in this model because only the low frequency behavior in the range $100 \mathrm{~Hz}$ to few $10 \mathrm{kHz}$ has to be studied. The transformer is modeled by a magnetizing inductance $l_{\mu}$ and a transformer ratio $m(m=100=n 2 / n 1) . r_{7}$ is the resistance of the secondary winding, $R_{2}$ is the load resistance (substituted later by the correcting dipole). The Hall sensor is modeled by a currentvoltage converter connected in series with the magnetizing inductance and the gain $\mathrm{K}$ determines the sensitivity of the Hall sensor. The Hall sensor acts as a $1^{\text {st }}$ order low-pass filter and its cut-off frequency is around $30 \mathrm{kHz}$. It is modeled by the $\mathrm{r}_{1} \mathrm{c}_{1}$ network. $\mathrm{R}_{6}$ and $\mathrm{C}_{6}$ are connected at the output of the Hall generator in order to reduced the noise at high frequency. $\mathrm{R}_{8}$ represents the internal resistance $\left(\mathrm{R}_{8}=8 \Omega\right.$, measured value) of the open-loop buffer (BUF 634) that is used to deliver the output current.

The transfer function of the sensitivity factor $\frac{V_{S}}{I}$ is given by (2):

$$
\frac{V_{S}}{I}=\frac{\frac{R_{3}}{m}+\frac{r_{7}}{j m^{2} 1_{\mu} \omega}\left(\frac{R_{3}}{R_{2}} F(j \omega)-\frac{R_{3}}{m}\right) \frac{1}{1+\frac{r_{7}}{j m^{2} l_{\mu} \omega}+\frac{F(j \omega)}{j m l_{\mu} \omega}}}{1+\frac{R_{3}}{R_{2}}+\frac{R_{8}}{R_{2}}+\frac{R_{3}}{j m l_{\mu} \omega}\left(\frac{R_{3}}{m}-\frac{R_{3}}{R_{2}} F(j \omega)\right) \frac{1}{1+\frac{r_{7}}{j m^{2} l_{\mu} \omega}+\frac{F(j \omega)}{j m l_{\mu} \omega}}}
$$

with:

$$
F(j \omega)=\frac{K}{\left(1+j r_{1} c_{1} \omega\right)\left(1+j R_{6} C_{6} \omega\right)}
$$

The values of the different parameters of Fig. 4.b model are given in Table. 1. Some values have been obtained by measurements, others have been selected in specifications.

Table. 1: Values of the different parts of Fig. 4.b model.

\begin{tabular}{cccc}
\hline $\mathrm{L}_{\mu}=100 \mathrm{nH}$ & $\mathrm{c}_{1}=2 \mathrm{nF}$ & $\mathrm{R}_{2}=15 \Omega$ & $\mathrm{R}_{8}=8 \Omega$ \\
\hline $\mathrm{m}=100$ & $\mathrm{R}_{6}=1 \mathrm{k} \Omega$ & $\mathrm{R}_{3}=50 \Omega$ & $\mathrm{K}=0.146$ \\
\hline $\mathrm{r}_{1}=1 \mathrm{k} \Omega$ & $\mathrm{C}_{6}=4.7 \mathrm{nF}$ & $\mathrm{r}_{7}=1.84 \Omega$ & \\
\hline
\end{tabular}

\section{Experimental results of the single stage $A C T$}

\section{Frequency domain results}


Fig. 5 shows a comparison between measurements and simulations in the uncorrected device. The results are in a good agreement at low frequency, thus confirming the validity of the model.

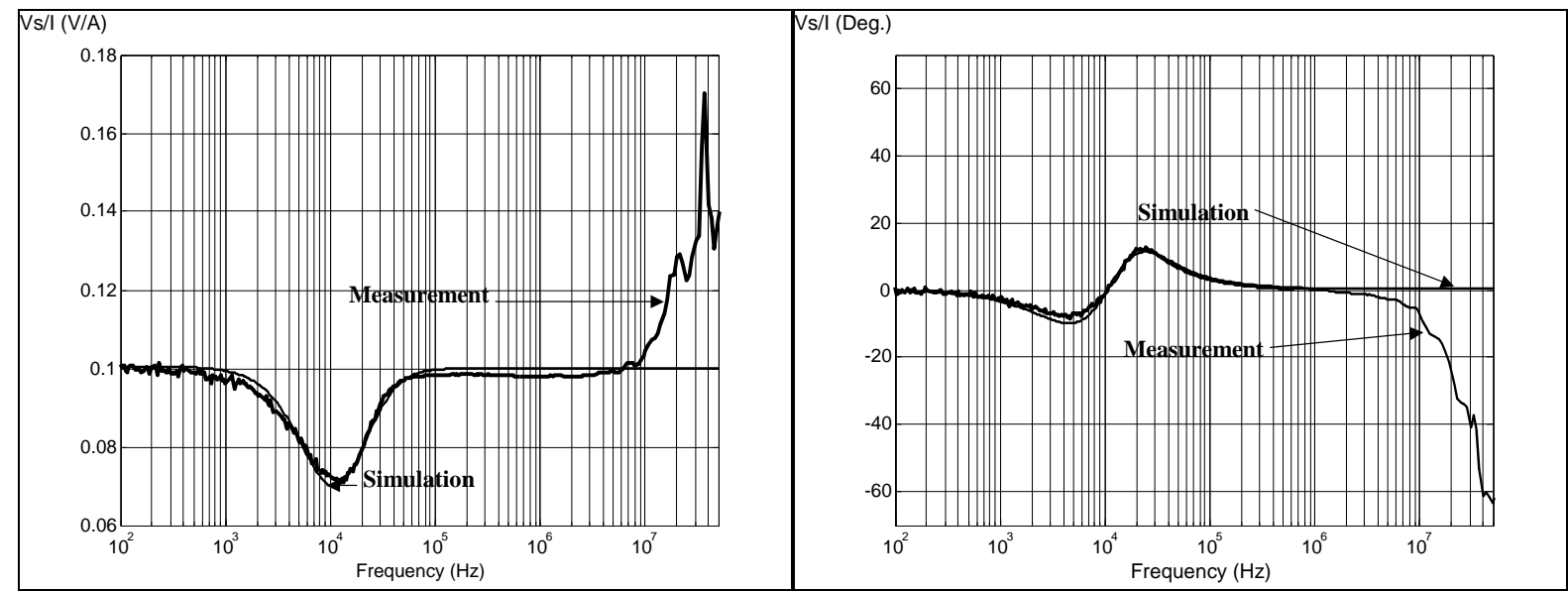

Fig. 5: Comparison between calculated and measured ACT transfer function.

Using the theoretical model, the elements of the correcting dipole (see Fig. 4.b) can be calculated in order to linearize the transfer function. The optimization routine has given the following values:

$$
\mathrm{R}^{\prime} 2=15 \Omega, \mathrm{C}_{10}=3.3 \mu \mathrm{F} \text { et } \mathrm{R}_{10}=2.91 \Omega .
$$

The correcting dipole has been included in the model and the new simulated transfer function curve $\frac{V_{S}}{I}$ is shown in Fig. 6. We can observe in this simulation that the curve is quite flat. The flatness error remains under $1 \%$, that can be compared to the $30 \%$ of Fig. 5 . Measurements are presented in Fig. 6, they have been acquired as presented in Fig. 1.b. Because the output current is small, the curve is noisy. Nevertheless, the linearity error is around $2 \%$, this confirms the modeling and the correcting principles. The small differences between simulations and measurements are due to the tolerance on the correcting capacitor $\mathrm{C}_{10}$, whose exact value has not been reached. However, over $10 \mathrm{MHz}$ the curves differ largely because the model is not matched with this frequency domain.

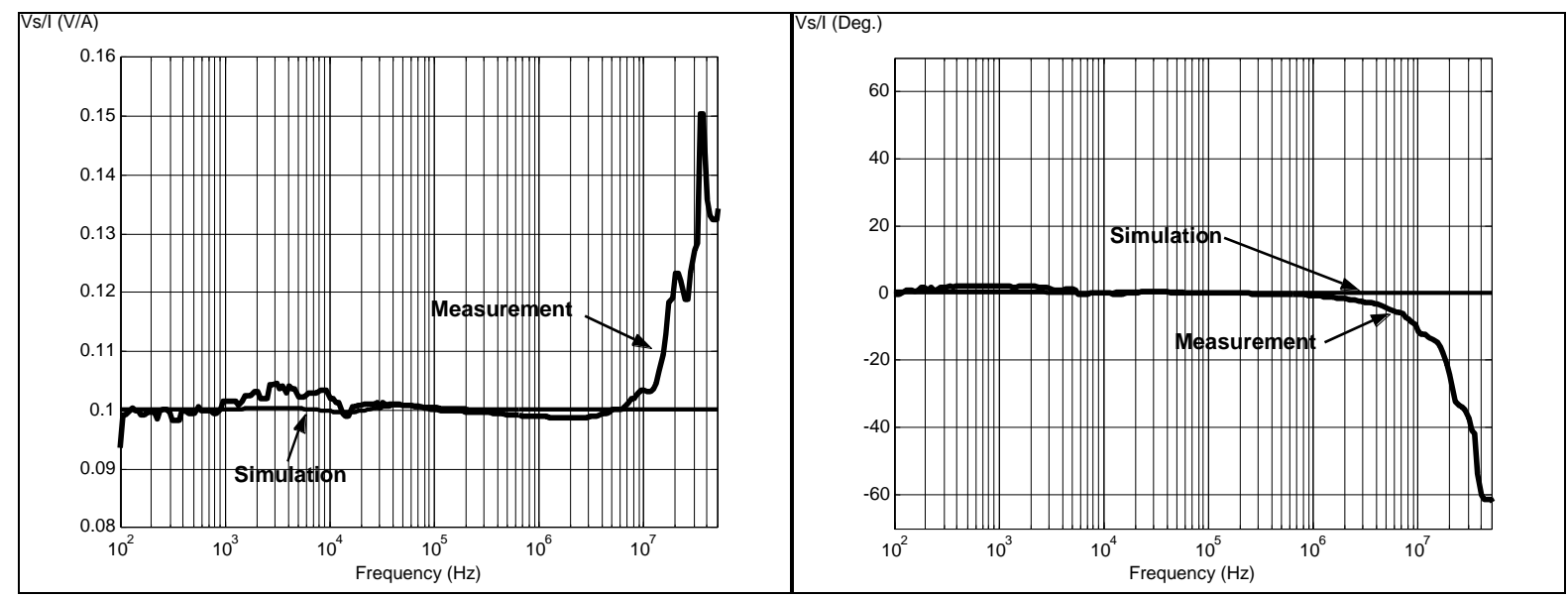

Fig. 6: Simulated and measured ACT corrected transfer function.

\section{Time domain results}


Fig. 7.a and Fig. 7.b shows the shape of the current flowing through the freewheeling diode of a $1 \mathrm{~kW}$ buck converter. When the switch opens the current in the load flows through the diode. After each transient, ringing occurs at about $10 \mathrm{MHz}$. This is due to the parasitic effects in the converter. Fig. 7.a shows the diode current measured with the ACT, and Fig. 7.b the market-standard current probe [4] measurement. Some differences after the negative slope can be observed in the market-standard current probe. It is probably caused by the current recovering transient of the amplifier.
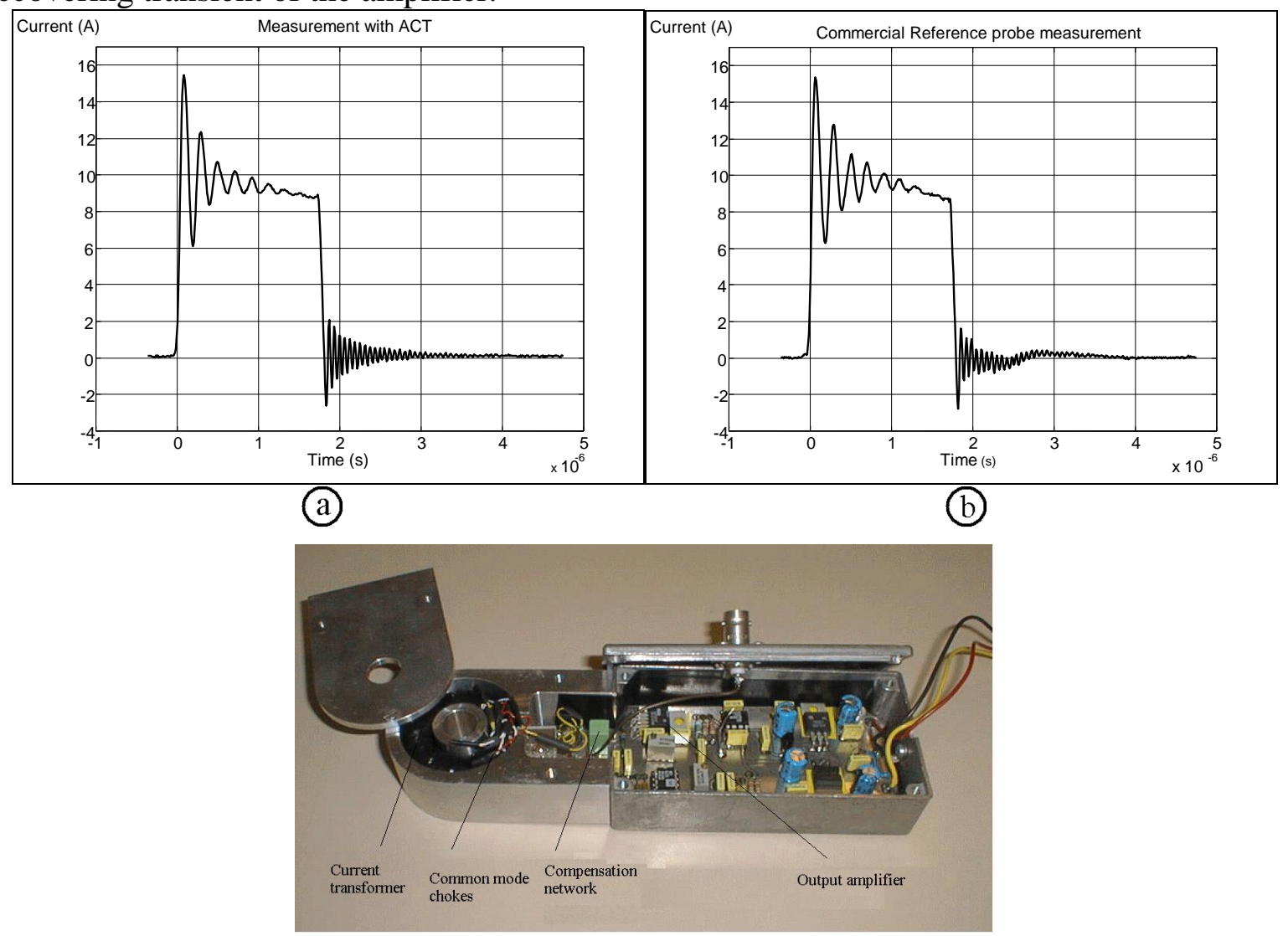

(c)

Fig. 7: (a) Current measurement with high dI/dt. (b) Comparison with a commercial device. (c) Picture of the ACT.

This experiment proves the efficiency of our device regarding other similar products. The design considerations and the correcting process are satisfactory.

\section{E. Single stage ACT realization}

Fig. 7.c shows a picture of the prototype of the single-stage ACT. A number of improvements have been realized in order to extend the high cut-off frequency.

- A multi-winding arrangement associated to common mode chokes at the ends of the windings has been used to minimize the turn-to-turn capacitance [5]. This feature increases the high cut-off frequency to around $30 \mathrm{MHz}$.

- A shielding case is placed around the current transformer in order to improve the immunity. All the electronics parts are placed in a metal case to allow a good behavior at high frequency. The ground of the supply is connected to the metal case.

- Precise capacitor $(2 \%)$ is used as $\mathrm{C}_{10}$. 


\section{IV.Double stage active current transformer: The $\mathrm{ACT}^{2}$}

\section{A. Double stage ACT principle}

The main limitation of the ACT is the high cut-off frequency. The frequency cut-off decreases for low values of the sensitivity factor $\left(\mathrm{V}_{\mathrm{s}} / \mathrm{I}\right)$. The sensitivity factor is inversely proportional to the transformer turn ratio. When the transformer turn ratio increases (i.e. the secondary number of turns increases) the parasitic capacitance of the winding increases too, limiting the high cut-off frequency. In order to overcome this limitation, a new double stack structure has been proposed in order to keep the same sensitivity while drastically reducing the total turns number. The principle is presented in Fig. 8.a. A single stage ACT transformer ratio of $\mathrm{m}=\mathrm{N}$, gives the same sensitivity factor value than a two-stage ACT when:

$$
m=m_{1} \times m_{2} \text {, with: } m_{1}=N_{1} \text { and } m_{2}=N_{2}
$$

Consequently, in the first case number of turns is $\mathrm{N}$ when it is $\mathrm{N}_{1}+\mathrm{N}_{2}$ in the second case. The total number of turns is drastically reduced, as the parasitic capacitance and the total stray inductances. Finally, the high cut-off frequency is improved.

\section{B. Double-stage transformer}

Suppose that sensitivity of the current transformer versus frequency acts as a low-pass filter, the upper frequency limit of a current transformer depends on the parasitic capacitances $\mathrm{C}_{\mathrm{s} 1}$ $\mathrm{C}_{\mathrm{s} 2}$ and on the total leakage inductance $\mathrm{l}_{\mathrm{f}}$. In the case of a single stage current transformer, the high cut-off frequency can be evaluated by (5).

$$
f_{c 1}=\frac{1}{2 \pi \sqrt{m^{2} l f C s}}
$$

In our case, because of multi-winding and common mode chokes, If $\approx 10 \mathrm{nH}$ and $C s \approx 0.3 \mathrm{pF}$, so $f_{c} 1 \approx 30 \mathrm{MHz}$. Assume that the parasitic capacitance depends proportionally on the secondary turns number, we get:

$$
C_{S 1}=C_{S} \frac{m_{1}}{m} \text { and } C_{S 2}=C_{S} \frac{m_{2}}{m}
$$

So, in the case of a double stage current transformer, the upper frequency limit is calculated by (7). In our case, $m_{1}=10$ and $m_{2}=30$, so $f_{c 2} \approx 90 \mathrm{MHz}$.

$$
f_{c 2}=\frac{1}{2 \pi \sqrt{m^{2} l f C_{S}\left(\frac{1}{m_{1}}+\frac{1}{m_{2}^{3}}\right)}}
$$

Compared to (5), (7) shows that the upper frequency limit is increased for the two-stage ACT, the frequency ratio is $\frac{f c_{1}}{f c_{2}}=\sqrt{m_{1}}$.

\section{Modeling of the $A C T^{2}$}

In this section the determination of the $\mathrm{ACT}^{2}$ model is proposed. The components values that ensure a trough cancellation of the transfer function, given by the Hall generator stage and the two-stage current transformer, are determined. Fig. 8.a shows the new model that is deduced 
from the single-stage ACT one. For clarity reasons, the details of the Hall sensor stage (depicted in Fig. 8.b) have not been represented.

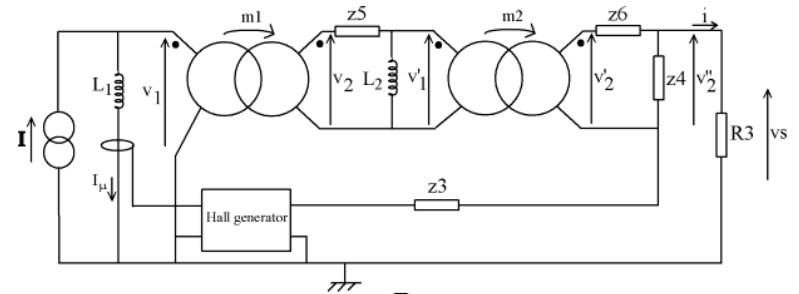

(a)

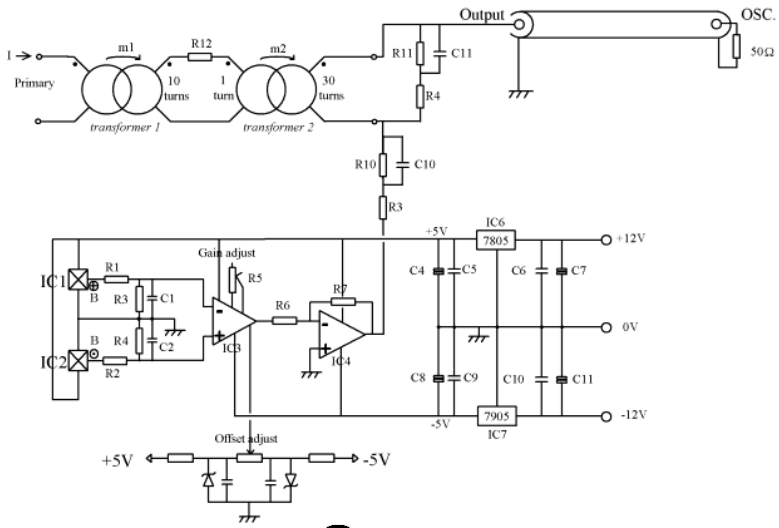

Fig. 8. (a) Two stages ACT model

(b) Electrical circuit of the ACT2 .

$\mathrm{L}_{1}$ : magnetizing inductance of the first stage current transformer

$\mathrm{m}_{1}$ : turn ratio of the first stage current transformer

$\mathrm{z}_{5}$ : first transformer secondary winding impedance

$\mathrm{L}_{2}$ : second transformer magnetizing inductance

$\mathrm{m}_{2}$ : second transformer turn ratio

$\mathrm{Z}_{6}$ : second transformer secondary winding impedance

$\mathrm{Z}_{4}$ : load resistance of the secondary winding

$\mathrm{Z}_{3}$ : output impedance of the amplifier

$\mathrm{R}_{3}$ : output load impedance

The values used in model of the Fig. 8.a are indicated in Table. 2. $\mathrm{L}_{1}, \mathrm{~L}_{2}$ are measured thanks to a Network Analyzer. z4 and z6 will be determined using an optimization algorithm.

Table. 2: parameter values of Fig. 8.a model.

\begin{tabular}{ccccccc}
\hline $\mathrm{L}_{1}$ & $\mathrm{~L}_{2}$ & $\mathrm{~m}_{1}$ & $\mathrm{~m}_{2}$ & $\mathrm{Z}_{5}$ & $\mathrm{Z}_{6}$ & $\mathrm{R}_{3}$ \\
\hline $43 \mathrm{nH}$ & $475 \mathrm{nH}$ & 10 & 30 & $0.112 \Omega$ & $0.23 \Omega$ & $50 \Omega$ \\
\hline
\end{tabular}

Referring to the $\mathrm{ACT}^{2}$ model of Fig. 8.a, the transfer function Vs/I is calculated by:

$$
V_{s} / I=\frac{1}{\frac{A \cdot B}{K . C}+\frac{z_{4}}{R_{3}\left(z_{4}+z_{6}\right)}\left(m_{1} \cdot m_{2}+m_{2} \frac{z_{5}}{j m_{1} L_{1} \omega}\right)}
$$

$\mathrm{K}$ is the value of the sensitivity factor of the Hall generator. $\mathrm{A}, \mathrm{B}$ and $\mathrm{C}$ are given by the following equations:

$$
\begin{gathered}
A=\frac{1}{(j \omega) m_{1} m_{2} L_{1}}+\frac{1}{(j \omega) \frac{m_{2}}{m_{1}} L_{2}}+\frac{z_{5}}{(j \omega)^{2} m_{1} m_{2} L_{1} L_{2}}+\frac{m_{1} m_{2}}{z_{4}+z_{6}}+\frac{m_{2} z_{5}}{z_{4}+z_{6}} \cdot \frac{1}{(j \omega) m_{1} L_{1}} \\
B=1+\frac{z_{3}}{R_{3}}+\frac{z_{4}}{R_{3}}-\frac{z_{4}{ }^{2}}{R_{3}\left(z_{4}+z_{6}\right)}-\frac{K \cdot z_{4}}{R_{3}\left(z_{4}+z_{6}\right)} \cdot \frac{z_{5}}{(j \omega) \frac{m_{1}}{m_{2}} L_{1}} \\
C=\frac{1}{(j \omega) m_{1} m_{2} L_{1}}+\frac{z_{5}}{(j \omega)^{2} m_{1} m_{2} L_{1} L_{2}}+\frac{z_{5}}{z_{4}+z_{6}} \cdot \frac{1}{(j \omega) \frac{m_{1}}{m_{2}} L_{1}}+\frac{1}{K} \frac{z_{4}}{z_{4}+z_{6}}
\end{gathered}
$$


Compared to the single stage current transformer (see Fig. 5), Fig. 9 shows that the trough is more important for the $\mathrm{ACT}^{2}$. So the correcting device will be constituted by two dipoles.
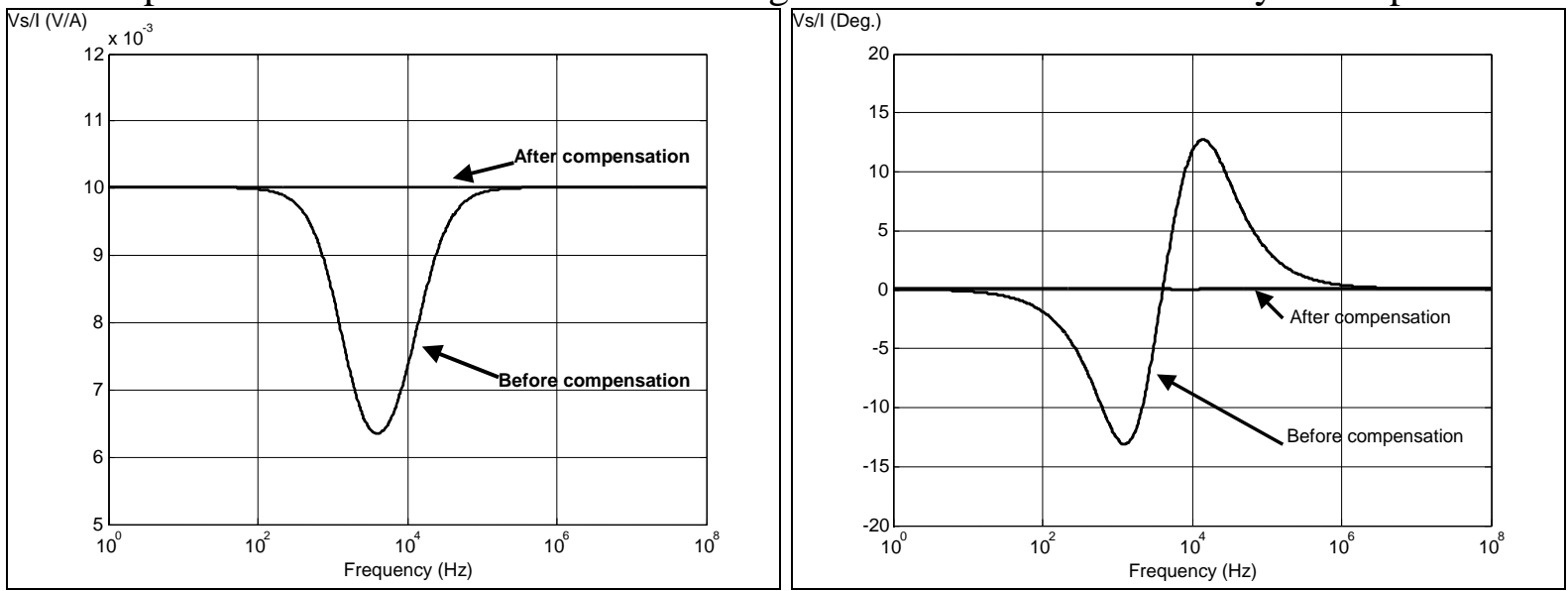

Fig. 9: Simulation of the transfer function of the double-stage ACT, modulus and phase.

\section{Designing of a double stage ACT}

\section{Specification of the double-stage ACT}

Our aim is to realize a $100 \mathrm{~A}, \mathrm{DC}$ to $50 \mathrm{MHz} \mathrm{ACT}^{2}$. Its sensitivity factor is chosen equal to 10 $\mathrm{mV} / \mathrm{A}$. The device must be shielded to achieve the EMI immunity required in power electronics applications. Fig. 8.b shows the electric circuit of the $\mathrm{ACT}^{2}$. In order to compensate the trough in the sensitivity curve, the two impedances $\mathrm{z}_{3}$ and $\mathrm{z}_{4}$ of Fig. 8. a model have been replaced by resistances $R_{3}, R_{4}, R_{10}, R_{11}$ and capacitances $C_{10}$ and $C_{11}$. These components constitute the correcting network. Table 4 gives the dimensions of the magnetic cores and the two transformers turns numbers.

Table. 3: Specifications of the two transformers.

\begin{tabular}{ccc}
\hline & Transformer 1 & Transformer 2 \\
\hline Outer diameter & $25 \mathrm{~mm}$ & $12 \mathrm{~mm}$ \\
Inner diameter & $16 \mathrm{~mm}$ & $8 \mathrm{~mm}$ \\
Height & $9 \mathrm{~mm}$ & $5 \mathrm{~mm}$ \\
Permeability $\mu$ & 4000 & 200 \\
Air-gap & $2 \mathrm{~mm}$ & none \\
Turns number & 10 & 30 \\
\hline
\end{tabular}

\section{Determination of the correcting networks}

The Hall sensor and the transformers cross-over operating frequency range is within the frequency range $100 \mathrm{~Hz}$ and $100 \mathrm{kHz}$, so networks $\mathrm{z}_{3}$ and $\mathrm{z}_{4}$ are calculated in order to correct the linearity error in this particular frequency range. All values are calculated by an optimization routine as previously presented [3], aiming to meet a linearity error smaller than $1 \%$. Six components values and the gain $\mathrm{K}$ value (determined by the sensitivity of the Hall generator and by the gain of the amplifier stage IC3 and IC4) have to be calculated. The optimization routine has to take into account some technological constraints that limit the value range of the components. For instance, the maximal output voltage of the amplifier 
limits the maximal values of $\mathrm{R}_{3}$ and $\mathrm{R}_{4}$ up to $100 \Omega$. Consequently, these component values have been imposed as constraints to the optimization routine.

The DC sensitivity factor value of the $\mathrm{ACT}^{2}$ is fixed by the network $\mathrm{z}_{3}$ and gain $\mathrm{K}$. Resistors $\mathrm{R}_{3}$ and $\mathrm{R}_{4}$ determine the sensitivity value in the high-frequency range. From a technological point of view, it is better to get the smallest capacitance values as possible and consequently the largest values for the resistors. Nevertheless, the values of capacitors $C_{10}$ and $C_{11}$ are situated in the micro-Farad order of magnitude. Practically, resistances $R_{10}$ and $R_{11}$ are adjusted in order to compensate the mismatched value of $\mathrm{C}_{10}$ and $\mathrm{C}_{11}$.

The optimized values of components that realize the optimal smoothing of the transfer function curve are given in Table 4. Note in Fig. 8.b that $\mathrm{R}_{12}$ is added in order to simplify the trough cancellation in transfer function.

Table 4 : Optimized values of the two correcting networks.

\begin{tabular}{cccccccc}
\hline $\mathrm{K}$ & $\mathrm{R}_{10}$ & $\mathrm{C}_{10}$ & $\mathrm{R}_{11}$ & $\mathrm{C}_{11}$ & $\mathrm{R}_{3}$ & $\mathrm{R}_{4}$ & $\mathrm{R}_{12}$ \\
\hline 0.0327 & $90 \Omega$ & $0.88 \mu \mathrm{F}$ & $1.27 \Omega$ & $3.74 \mu \mathrm{F}$ & $23.5 \Omega$ & $4.69 \Omega$ & $0.1 \Omega$ \\
\hline
\end{tabular}

Fig. 9 shows the transfer function of the $\mathrm{ACT}^{2}$ simulated by (8), modified in order to take into account the correcting network. Before compensation, the linearity error was around $35 \%$ and this value is reduced to around $0.4 \%$ with the correcting networks.

\section{E. Experimental results}

\section{Frequency domain results}

Fig. 10 shows a comparison of the transfer function of the $\mathrm{ACT}^{2}$ described herein and a market-standard current probe [4]. The smoothing error of the $\mathrm{ACT}^{2}$ transfer function is situated in the range $+2 /-6 \%$ while the market-standard current probe is situated around $6 \%$. The results are close together and prove the efficiency of our device. Nevertheless, the linearity error is not as great as expected. This difference is due to the inaccuracy of the capacitors used in the correcting networks, as a sensitivity calculation has shown to us. Finally, the linearity error of the $\mathrm{ACT}^{2}$ is about $8 \%$ and its high cut-off frequency is close to $50 \mathrm{MHz}$.

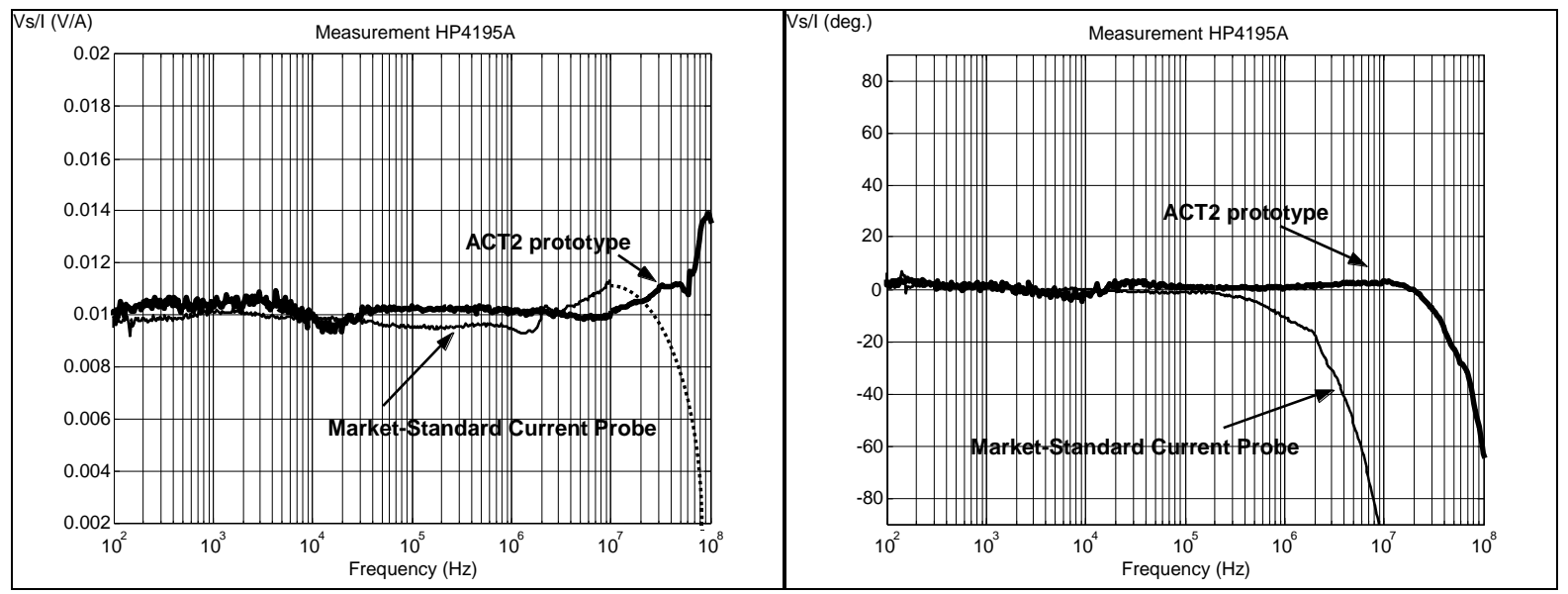

Fig. 10: Comparison between the $\mathrm{ACT}^{2}$ and a market-standard current probe.

\section{Time domain results}

Fig. 11 shows the current measurement diagram of an IGBT tested under short-circuit conditions [9]. The current reaches $100 \mathrm{~A}$ and a high frequency (around $200 \mathrm{kHz}$ ) ringing (50 
A) is superposed. The current measurement of $\mathrm{ACT}^{2}$ is compared to a commercial current transducer [1]. Some significant differences can be noticed because the commercial current probe has a limited output signal dynamic. Voltage $\mathrm{V}_{\mathrm{gs}}$ monitors exactly the IGBT current when it is on. During ringing phase, ringing peaks in $\mathrm{V}_{\mathrm{gs}}$ keep a quasi-constant amplitude that is attested by $\mathrm{ACT}^{2}$ probe but not correctly measured by the commercial probe. Concerning the $\mathrm{ACT}^{2}$, a small voltage drift just after the negative slope can be noticed, it is caused by the linearity drop of $6 \%$ in the transfer function as shown previously.

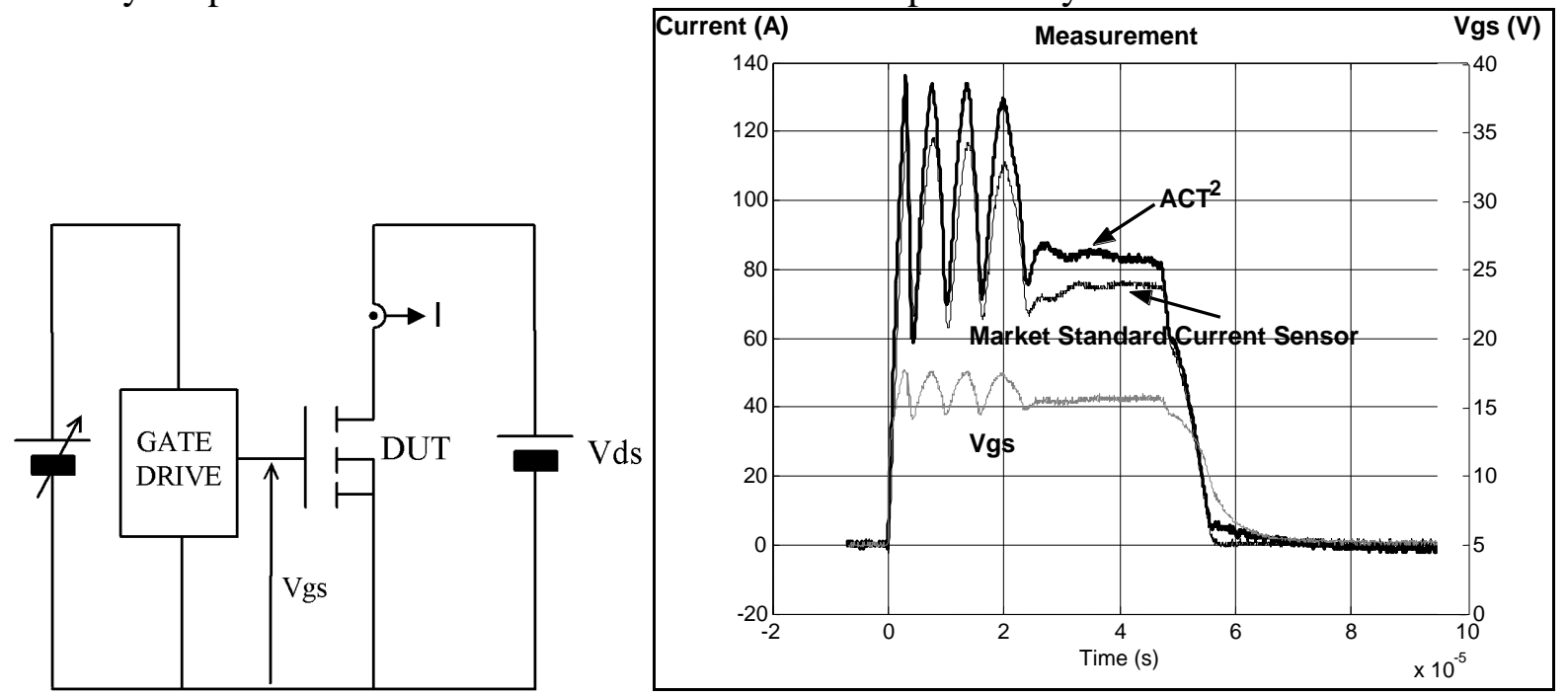

Fig. 11: Measurement diagram and current measurements comparison.

\section{F. Practical building}

Fig. 12.a shows a picture of the $\mathrm{ACT}^{2}$. Note the shielding case that protects against outer electromagnetic fields. It has also a beneficial effect on the $\mathrm{ACT}^{2}$ electrical performances because it reduces the inner leakage inductance and it limits the capacitive couplings between the primary and the secondary windings and between the two transformers. A common mode choke in the power supply wire is crucial for avoiding extra capacitive couplings. Without it, the increase of transfer function level at $100 \mathrm{MHz}$ has been measured around $25 \mathrm{~dB}$. Moreover, it is important to reduce the capacitive couplings between the electronics and the primary conductor. So the electronic card is shielded against capacitive coupling.

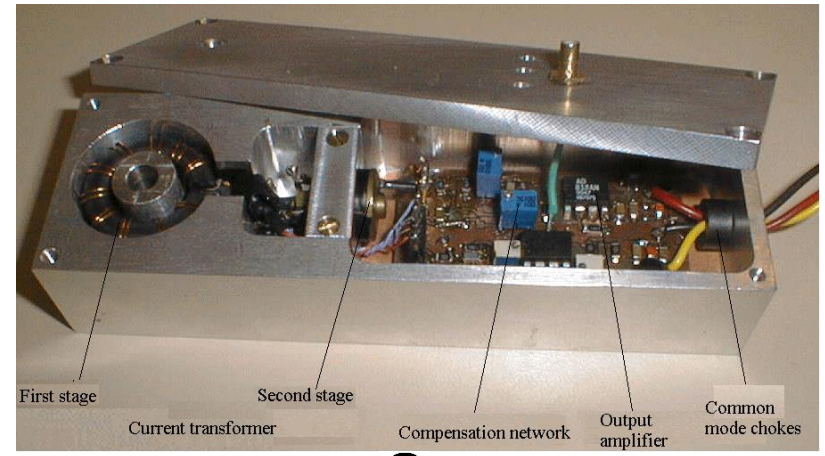

(a)

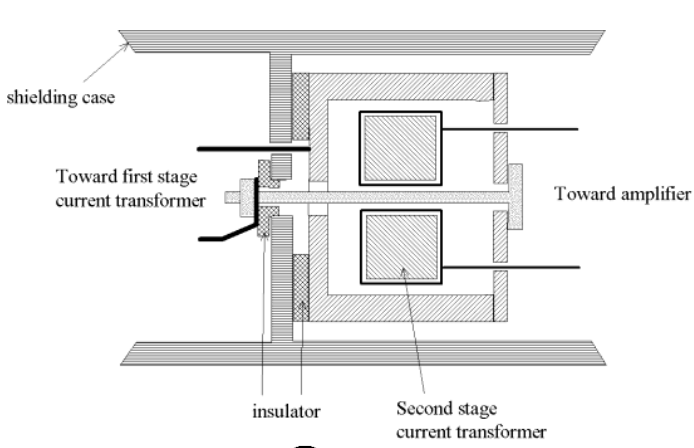

(b)

Fig. 12: (a) Picture of the $\mathrm{ACT}^{2}$. (b) Detail of the $\mathrm{ACT}^{2}$ second stage. 
Fig. 12.b shows a detail of the second stage current transformer. A special insulated coaxial arrangement is used to reduce parasitic capacitive couplings between the shield and the second stage. In that way, the ends of the current transformer are not connected to ground, and the output voltage is not disturbed by the common mode current that flows through the shield. Moreover, this configuration makes the magnetic coupling between the axial conductor and the secondary winding symmetric, leading to reduce the leakage inductance and thus improving the high frequency bandwidth as Fig. 10 shows.

\section{Conclusion}

This paper has presented the interest of the active current probe (ACT) versus the classical closed-loop current sensor. A single-stage ACT has been modeled and designed. Its limitations have been highlighted. A correcting networks has been proposed to overcome the linearity error in the cross-over frequency ranges of the Hall sensor and the current transformer. Furthermore, a new two-stage ACT has been proposed in order to overcome the previous ACT limitations in high frequency range.

Two prototypes have been realized and their performances have been presented in the time and frequency domains. Some practical improvements have been made that increase the bandwidth and/or the EMI ruggedness. These devices are particularly well suited to power electronics measurements which require improved dynamic performances, high current gauge and EMI compatibility.

\section{References :}

[1] LEM, "Isolated current and voltage transducers", $3^{\text {rd }}$ Edition. 2004. [Online]. Available: http://www.lem.com.

[2] HONEYWELL, "Interactive catalog from Honeywell for SS495A1 Hall sensor". 2004.

[Online]. Available:

http://content.honeywell.com/sensing/prodinfo/solidstate/catalog/c20020.pdf

[3] Matlab from MathWorks with optimization toolbox.

[4] Tektronix Instruction manual for Current Probe Amplifier type AM 503 with A6302 Probes.

[5] F. Costa, E. Labouré, F. Forest, and C. Gautier, "Wide bandwidth, large AC current probe for power electronics and EMI measurements", IEEE Trans. On Industrial Electronics, vol. 44, $\mathrm{N}^{\circ} 4$, pp 502-511, August 1997.

[6] E. Labouré, F. Costa and F. Forest, "Current measurement in static converters and realization of a high frequency passive current probe (50A-300MHz)" in Proc. EPE'93, Brighton, U.K., $\mathrm{N}^{\circ}$ 377, vol. 4, $\mathrm{N}^{\circ}$ 377, pp 478-783, 1993.

[7] F. Costa, P. Poulichet, F. Mazaleyrat, E. Labouré, "The Current Sensors in Power Electronics, a Review", EPE Journal, N 1, Vol. 11, pp. 7 - 18, 2001.

[8] P. Poulichet, F. Costa, É. Labouré, "High-Frequency Modeling of a Current Transformer by Finite-Element Simulation", IEEE Transactions on Magnetics, N 2, Vol. 39, pp 998-1007, Mars 2003.

[9] F. Saint-Eve, S. Lefebvre, Z . Khatir, "Influence of repetition of short-circuits conditions on IGBT lifetime", Conf. EPE (Toulouse) 2003.

[10] SCHOTT C., BLANCHARD H., POPOVIC R. S., RACZ R., HREJSA J., "HighAccuracy Analog Hall Probe", IEEE Transactions on Instrumentation and Measurement, pp 613-616, 1997. 
[11] ETTER M. , FRIOT M., "Wide bandwidth, Accurate current and Voltage Transducers" PCIM Europe Nov./Dec. 1993.

[12] GROENENBOOM M., LISSER J., "The Zero-Flux Current Transformer a High Precision Wide-Band Measuring Device" IEEE Trans. on Nuclear Science, 1977. 